\title{
Segmentation of Textile Textures using Contextual Clustering
}

\author{
Shobarani \\ Research Scholar, \\ Department of Computer Science, \\ Mother Teresa Women's University, \\ Kodaikanal, India-624 101.
}

\author{
Dr.S.Purushothaman \\ Dean, Udaya School of Engineering \\ Vellamodi, Ammandivilai, \\ India-629204.
}

\begin{abstract}
This paper presents texture segmentation concept using supervised method in contextual clustering and fuzzy logic. The data set used is the textile textures. The image is split into $3 \times 3$ windows. The features of the windows are presented to the input layer of the contextual clustering. The algorithm involves least computation in the segmentation of textures. The output of fuzzy logic depends upon the radii of the clusters used during segmentation. The implementation of the algorithm is made by the fuzzy membership its probability indicates the spatial influence of the neighboring pixels on the centre pixel.
\end{abstract}

\section{Keywords}

Image segmentation, clustering, fuzzy logic, textures.

\section{INTRODUCTION}

In this research work, the investigations are carried out on textile textures and mosaic using fuzzy logic, contextual clustering for improved texture segmentation. Texture is the result of spatial variation in pixel intensities in an image. Texture is useful in a variety of applications and has been a subject of intense study by many researchers. Recognition of homogeneous image regions using texture properties is an important application. The goal is to do classification of input image with the existing textures template. Texture segmentation is achieved using texture properties.

\section{PREVIOUS WORK}

Yining Deng et. al., (2001) proposed a class map method for texture segmentation[10]. Applying the criterion to local windows in the class-map results in the image, in which high and low values correspond to boundaries and interiors of colortexture regions. A region growing method is then used to segment the image based on the multiscale J-images. Nikos Paragios (2002) presented variational framework for boundary and region-based segmentation under a curve-based optimization objective function[5]. The task of supervised texture segmentation is considered to demonstrate the potentials of the proposed framework. Arivazhagan et. al., (2003) describes multiple scales based on block by block comparison of wavelet co-occurrence features with template features[1,2]. Sara et. al., (2006) use Uniform Local Binary Pattern (ULBP) method to extract texture features[6]. Image segmentation is carried out using the K-means algorithm on feature vectors, including color and texture features. The distance measure is defined as a function of the color and texture feature vector distances from the K-means defined as centers. The weighting parameter is used to adjust the relative contribution of the color and texture features. The proposed algorithm is applied to color images in the (Red, Green, Blue -RGB), (Hue, Saturation, Value-HSV) and (Improved Hue, Saturation, and Lightness-IHLS) color spaces. [8]Soo et, al., (2007) extracts texture features using the wavelet packet frame decomposition followed by a Gaussianmixture-based classifier, which assigns each pixel to a class. This scheme shows high recognition accuracy in the classification of Brodatz texture images. Lin Ma et. al., (2009) proposes Ant Colony Optimization (ACO) for texture segmentation[3]. The study uses the direct thresholding method, which is based on wavelet transform smooth sub-images from the use of a quadrant mean filtering method, to attenuate the texture background and preserve the anomalies. The images are then segmented by threshold processing and noise filtering. [7]Shoudong Han et. al., (2011) proposes a novel texture segmentation approach using independent-scale component-wise Riemannian-covariance Gaussian mixture model (ICRGMM) in Kullback-Leibler (KL) measure based multi-scale nonlinear structure tensor (MSNST) space.

\section{SCOPE OF PRESENT WORK}

In this work, a systematic approach has been developed to extract textures from the given texture images. The features are extracted using contextual Clustering. The extracted features are used to segment the image using supervised method, fuzzy logic. The major combinations of the algorithms developed are as follows:

(i) Contextual clustering using supervised method

(ii) Fuzzy logic using supervised method.

\section{SIMULATION OF DATA}

\subsection{Data Collection}

A sample database has been presented in Table 1. The table presents few textile textures. 
Table 1 Sample Database.

The table1 presents few textile textures
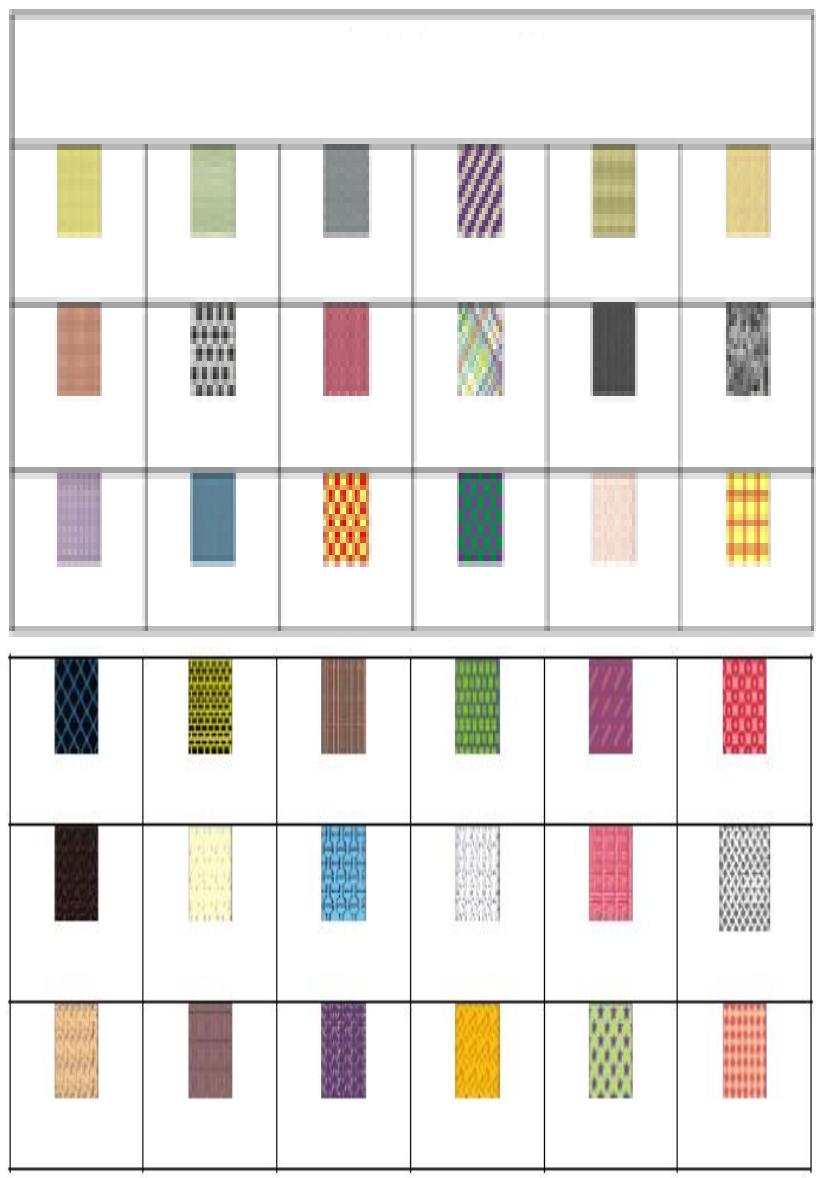

In this paper, for evaluating the proposed technique, here used two image databases that are called Tilda and Brodatz. Tilda includes 8 groups of different fabric textile types and Each group has 50 images with 512 x 768 pixels. Brodatz includes 112 types of fabric images with $640 \times 640$ pixels.

\subsubsection{Tilda}

Tilda was developed within working group Texture Analysis of the DFGs (Deutsche Forschungs gemeinschaft). It is a textile texture database. This analyzed methods made it possible to recognize and distinguish textures of varying kinds. In this database contains the total of eight representative kinds of textiles which is based on the analysis of textile atlases. There are 3200 TIF pictures with a storage capacity of 1.2 Gbyte in the entire textile texture database., ASCII file exists in every picture in the TILDA database.

\subsubsection{Brodtz}

For the Brodatz texture images, the page number of the corresponding texture in the Brodatz book is in parenthesis. The digital images from the texture book are not scanned the pages. The images are scans of a set of glossy black and white prints were purchased by the author. The same textures of pictures are prints as in the book, in most cases images are not same as the one in the book.

\section{ALGORITHMS PROPOSED FOR IMAGE TEXTURE SEGMENTATION}

\subsection{Contextual Clustering ( CC)}

Picture Segmentation is a subjective and context dependent cognitive process. In mathematical terms, this process is illposed. i.e. there is no single goal for picture partition algorithms. To make a picture segmentation is a well posed problem, it is regarded as an optimization task featuring a firm statistic foundation. Recently there has been considerable interest in statistical clustering techniques in image segmentation inspired by the methods of statistical physics, which were developed to study the equilibrium properties of large, lattice based systems consisting of identical interacting components. In a clustering technique for image segmentation each pixel is associated with one of the finite number of categories to form disjoint regions.

Contextual clustering algorithm segments a data into category 1 $\left(\omega_{0}\right)$ and category $2\left(\omega_{1}\right)$. They are assumed to be drawn from standard normal distribution. The following steps are adopted for implementing contextual clustering.

(i) Define decision parameter $\mathrm{T}_{\mathrm{cc}}$ (positive) and weight of neighborhood information $\beta$ (positive). Let $N_{n}$ be the total number of data in the neighborhood. Let $\mathrm{Z}_{\mathrm{i}}$ be the data itself, i.

(ii) Classify data with $z_{i}>T_{\alpha}$ to $\omega_{1}$ and data to $\omega_{0}$. Store the classification to $\mathrm{C}_{0}$ and $\mathrm{C}_{1}$.

(iii) For each data $\mathrm{i}$, count the number of data $u_{i}$, belonging to class $\omega 1$ in the neighborhood of data $i$. Assume that the data outside the range belong to $\omega_{0}$.

(iv) Classify data with

$$
\mathrm{z}_{\mathrm{i}}+\frac{\beta}{\mathrm{T}_{c c}}\left(\mathrm{u}_{\mathrm{i}}-\frac{\mathrm{N}_{\mathrm{u}}}{2}\right)>\mathrm{T}_{\mathrm{a}}
$$

Store the classification to variable $\mathrm{C}_{2}$.

(v) If $\mathrm{C}_{2} \neq \mathrm{C}_{1}$ and $\mathrm{C}_{2} \neq \mathrm{C}_{0}$, copy $\mathrm{C}_{1}$ to $\mathrm{C}_{0}, \mathrm{C}_{2}$ to $\mathrm{C}_{1}$ and return to step 3 , otherwise stop and return to $\mathrm{C}_{2}$.

The implementing of the $\mathrm{CC}$ is given as follows:

Step 1: Read a Pattern (texture image feature).

Step 2: Sort the values of the Pattern.

Step 3: Find the Median of the Pattern $\mathrm{Cm}$.

Step 4: Find the number of values greater than the Median Values, $\mathrm{U}_{\mathrm{m}}$. $(b s / 2))$.

Step 5: Calculate CC using $\mathrm{C}_{\mathrm{m}}+($ beta/Tcc $) *\left(\mathrm{U}_{\mathrm{m}}-\right.$

Step 6: Assign CC as the segmented values.

Figure 1 shows a steady increase in the error as the beta value changes from 0.1 to 1 . Hence, lower beta value is preferred for better estimation by $\mathrm{CC}$. 


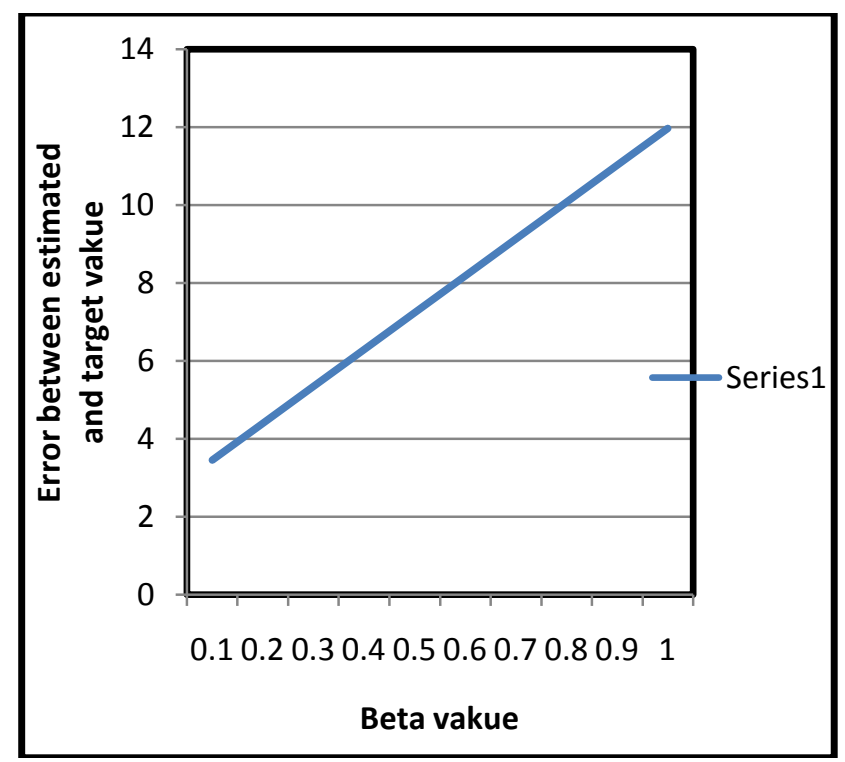

Fig. 1 Impact of Beta value in CC estimation

Figure 2 shows a steady decrease in the error as the threshold value changes from 10 to 100 . Hence, higher threshold value is preferred for better estimation by CC.

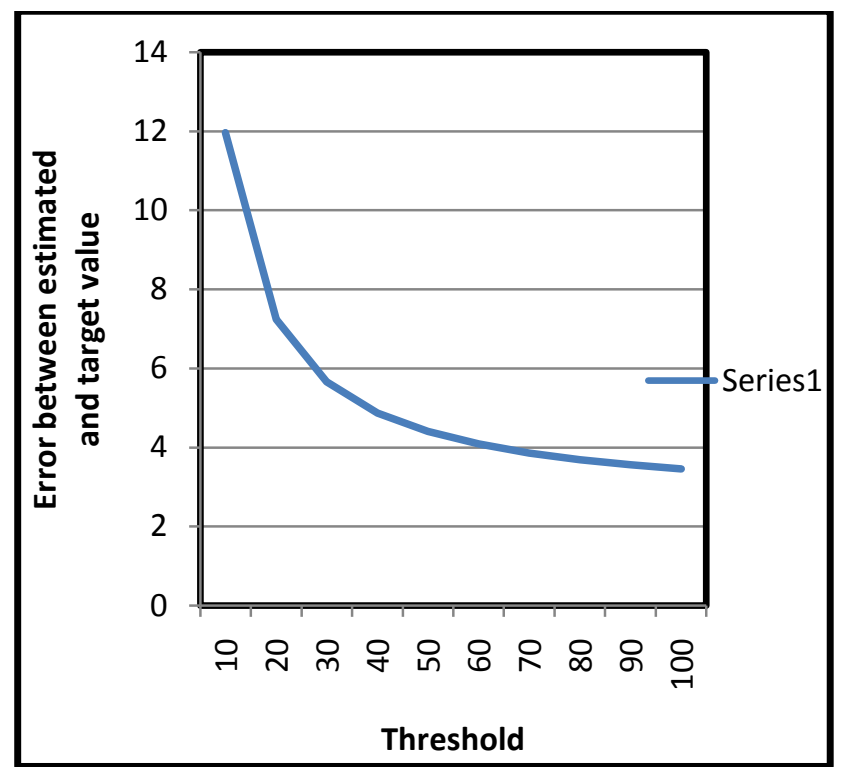

Fig. 2 Impact of Threshold value in CC estimation

The segmentation analysis is shown in Figure 3.
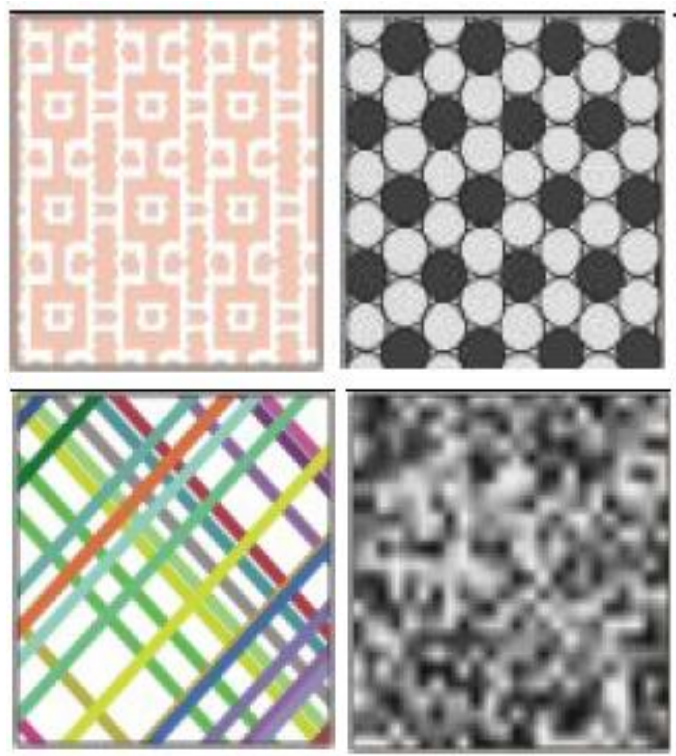

(a) Original Image

(240)

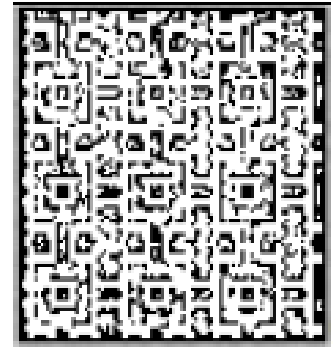

(225)

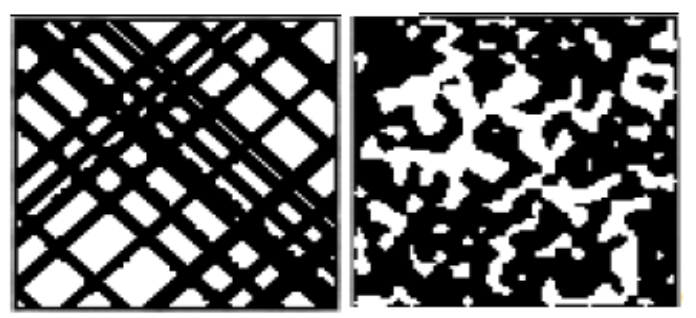

(b) CC segmented image
(210)

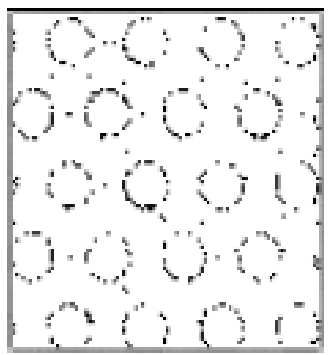

(230)

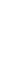



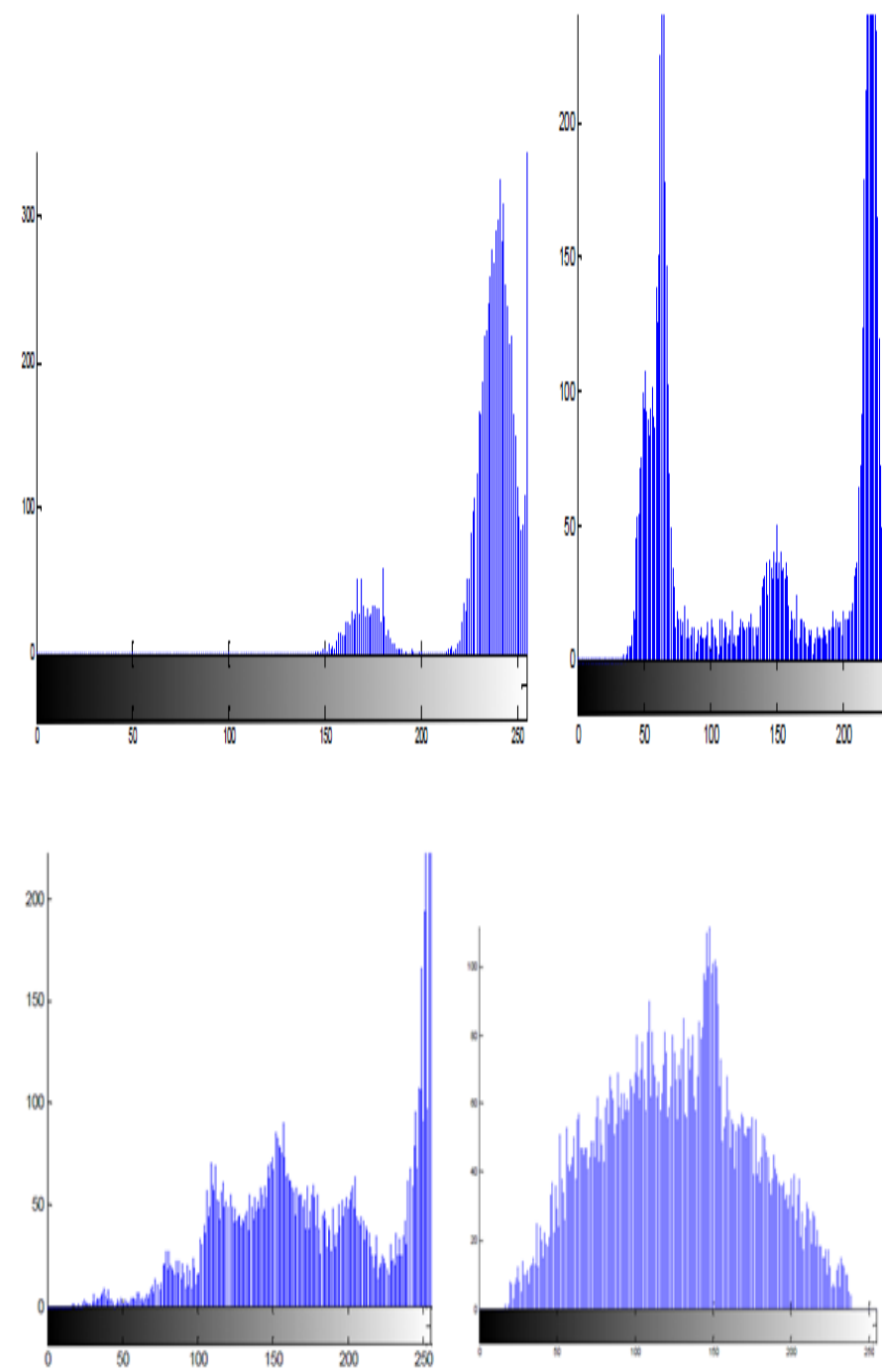

(c) Histogram

Fig. 3 CC segmentation analysis

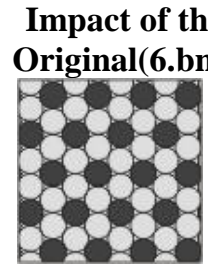

50

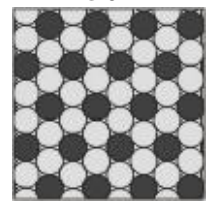

50

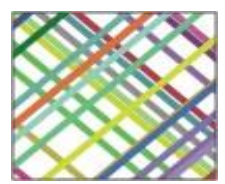

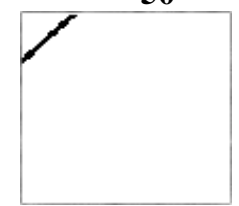

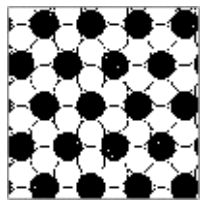

150

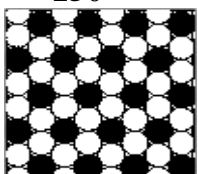

210

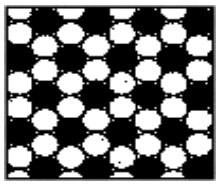

240
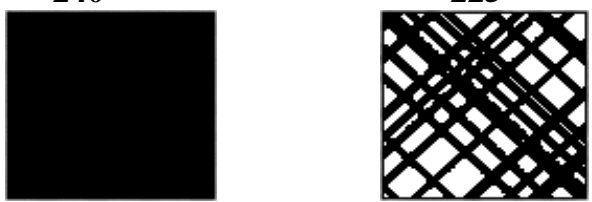

Fig. 4 Impact of threshold in segmentation

\subsection{Fuzzy Logic}

Weina Wang et al (2006) used the Many clustering strategies such as the crisp clustering scheme and the fuzzy clustering scheme, each of which has its own special characteristics[13]. Zadeh L.A.,(1965) proposed the conventional crisp clustering method restricts each point of the data set to exclusively just one cluster[14], which involves the idea of partial membership described by a membership function, Bezdek J.C, Ferahta N and Zhang D.Q used the fuzzy clustering as a soft segmentation method has been widely studied and successfully applied to image segmentation[16-22]. Bezdek J.C extracts among the fuzzy clustering methods, fuzzy $c$-means (FCM) algorithm[15] is the most popular method used in image segmentation because it has robust characteristics for ambiguity and can retain much more information than hard segmentation methods discussed by Bezdek J.C and Zhang D.Q et al[16,22]. Although the conventional FCM algorithm works well on most noise-free images, it has a serious limitation: it does not incorporate any information about spatial context, which cause it to be sensitive to noise and imaging artifacts. To compensate for this drawback of FCM, the obvious way is to smooth the image before segmentation. The training and testing fuzzy logic is to map the input pattern with target output data. For this, the inbuilt function has to prepare membership table and finally a set of number is stored. During testing, the membership function is used to test the pattern. 
Training Fuzzy logic

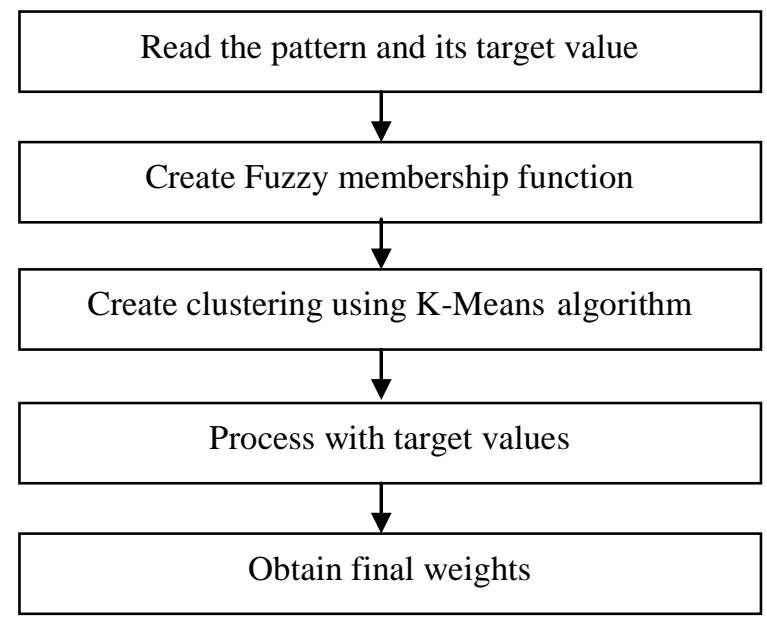

Fig. 5 Training Fuzzy Logic

Testing Fuzzy logic for texture segmentation

\begin{tabular}{|c|}
\hline Input a pattern \\
\hline Process with Fuzzy membership function \\
\hline Find the cluster to which the pattern belongs \\
\hline Obtain estimated target values \\
\hline
\end{tabular}

Fig. 6 Testing Fuzzy logic

Table 2 presents the segmented texture outputs for two different radii.

\begin{tabular}{|l|l|}
\hline Radii in Fuzzy logic & Segmented output \\
\hline 0.1 (Figure 7) \\
\hline 0.6 (Figure 8)
\end{tabular}

Table 2 Outputs of Fuzzy logic

RADII specifies the range of influence of the cluster center for each input and output dimension, assuming the data falls within a unit hyperbox (range [0 1]). Specifying a smaller cluster radius will usually yield more, smaller clusters in the data, and hence more rules. When RADII is a scalar it is applied to all input and output dimensions.

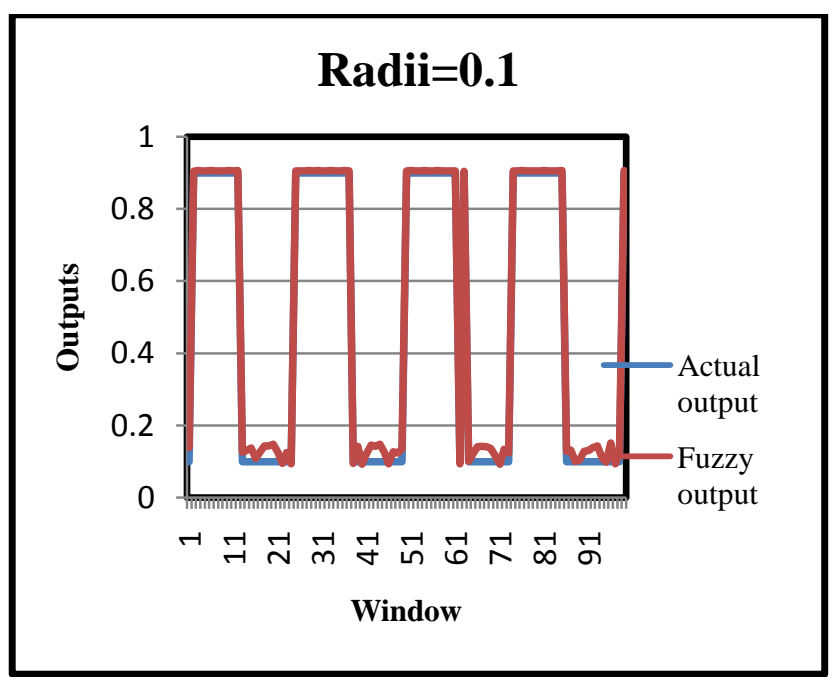

Fig. 7 Output of Fuzzy logic ( Radii=0.1)

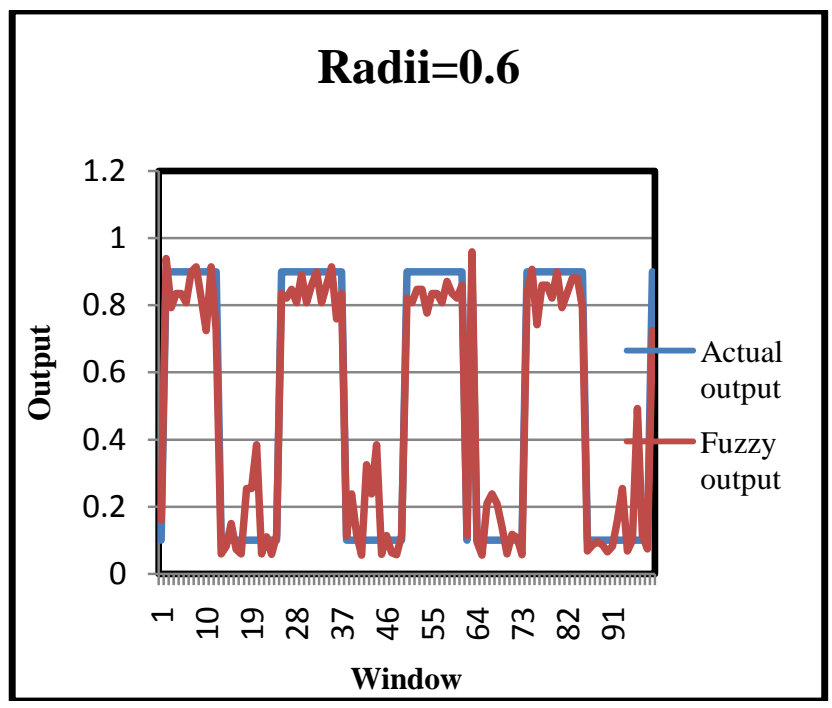

Fig.8 Output of Fuzzy logic (Radii= 0.6)

\section{CONCLUSIONS}

In this work, texture segmentation for textile textures and mosaic is considered. The conclusions of this research work are

(i) The supervised contextual clustering extracts features of the texture image which represents information in a given window. The algorithm involves least computation in the segmentation of textures.

(ii) The output of fuzzy logic depends upon the radii of the clusters used during segmentation.

In this work, suitable smoothing schemes have also been used to overcome some of the earlier reported limitations of the crisp clustering scheme. 


\section{REFERENCES}

[1] Arivazhagan S., Ganesan L., "Texture segmentation using wavelet transform", Pattern Recognition Letters, Volume 24 Issue 16, pp. $3197-3203,2003$.

[2] Arivazhagan S., Ganesan L., "Texture classification using wavelet transform", Pattern Recognition Letters, v.24 n.910, p.1513-1521, 2003.

[3] Lin Ma, Kuanquan Wang, David Zhang, "A universal texture segmentation and representation scheme based on ant colony optimization for iris image processing", Computers \& Mathematics with Applications, v.57 n.11-12, p.1862$1868,2009$.

[4] Mohand Saïd Allili, Djemel Ziou, "Globally adaptive region information for automatic color-texture image segmentation", Pattern Recognition Letters, v.28 n.15, p.1946-1956, 2007.

[5] Nikos Paragios , Rachid Deriche, "Geodesic Active Regions and Level Set Methods for Supervised Texture Segmentation", International Journal of Computer Vision, v.46 n.3, p.223-247, 2002.

[6] Sara Arasteh, Chih-Cheng Hung, "Color and texture image segmentation using uniform local binary patterns", Machine Graphics \& Vision International Journal, v.15 n.3, p.265-274, 2006.

[7] Shoudong Han, Wenbing Tao, Xianglin Wu, "Texture segmentation using independent-scale component-wise Riemannian-covariance Gaussian mixture model in KL measure based multi-scale nonlinear structure tensor space”, Pattern Recognition, v.44 n.3, p.503-518, 2011.

[8] Soo Chang Kim, Tae Jin Kang, "Texture classification and segmentation using wavelet packet frame and Gaussian mixture model", Pattern Recognition, Volume 40 Issue 4, pp.1207-1221, 2007.

[9] Won W. K. g, C. W. M. Yuen D. D. Fan L. K. Chan E. H. K. Fung, "Stitching defect detection and classification using wavelet transform and BP neural network", Expert Systems with Applications: An International Journal, Volume 36 Issue 2, 2009.

[10] Yining Deng, b.s. Manjunath, "Unsupervised Segmentation of Color-Texture Regions in Images and Video", IEEE Transactions on Pattern Analysis and Machine Intelligence, v.23 n.8, p.800-810, 2001.

[11] Workgroup on"Texture Analysis of DFG, "TILDA Textile Texture Database”, http://lmb.informatik.uni-freiburg.de/ research/dfg-texture/tilde

[12] P.Brodatz, Textures: A Photographic Album for Artists and Designers, Dover, New York, (1966)

[13] Weina Wang, Yunjie Zhang, Yi Li, Xiaona Zhang, The global fuzzy c-means clustering algorithm,[In] Proceedings of the World Congress on Intelligent Control and Automation, Vol. 1, 2006, pp. 3604-3607.

[14] Zadeh L.A., Fuzzy sets, Information and Control, Vol. 8, 1965, pp. 338-353.
[15] Bezdek J.C., Pattern Recognition with Fuzzy Objective Function Algorithms, Plenum Press, NewYork 1981.

[16] Bezdek J.C., Hall L.O., Clarke L.P., Review of MR image segmentation techniques using pattern recognition, Medical Physics 20(4), 1993, pp. 1033-1048. Image segmentation based on fuzzy clustering ... 147

[17] Ferahta N., Moussaoui A., Benmahammed K., Chen V., New fuzzy clustering algorithm applied to RMN image segmentation, International Journal of Soft Computing 1(2), 2006, pp. 137-142.

[18] Tolias Y.A., Panas S.M., On applying spatial constraints in fuzzy image clustering using a fuzzy rule-based system, IEEE Signal Processing Letters 5(10), 1998, pp. 245-247.

[19] Tolias Y.A., Panas S.M., Image segmentation by a fuzzy clustering algorithm using adaptive spatially constrained membership functions, IEEE Transactions on Systems, Man and Cybernetics, Part A 28(3), 1998, pp. 359-369.

[20] Noordam J.C., Van Den Broek W.H.A.M., Buydens L.M.C., Geometrically guided fuzzy C-means clustering for multivariate image segmentation, [In] Proceedings 15-th International Conference on Pattern Recognition, Vol. 1, 2000, pp. 462-465.

[21] Ahmed M.N., Yamany S.M., Mohamed N., Farag A.A., Moriarty T., A modified fuzzy C-means algorithm for bias field estimation and segmentation of MRI data, IEEE Transactions on Medical Imaging 21(3), 2002, pp. 193199.

[22] Zhang D.Q., Chen S.C., Pan Z.S., Tan K.R., Kernel-based fuzzy clustering incorporating spatial constraints for image segmentation, [In] Proceedings of International Conference on Machine Learning and Cybernetics, Vol. 4, 2003, pp. 2189-2192.

\section{AUTHORS PROFILE}

Shoba Rani received the MCA degree from Bharathidasan University. She worked as a Lecturer in LWIASE, Chennai. Also, she completed M.Phil. from Annamalai University. After that she worked for two years as a Consultant in Computer Science Dept. for TamilNadu Open University, Chennai. Presently she is working as a Assist.Prof in Dr. MGR University, Chennai. She is also a Ph.D. research scholar in the department of Computer Science at Mother Teresa Women's University, Kodaikanal.

Dr. S. Purushothaman, is presently working as Dean, Udaya School of Engineering, Vellamodi, Ammandivilai, Nagercoil. He is obtained his Ph.D. degree from IIT(M), Chennai. He has so far successfully guided nearly Ten Ph.D. Research Scholars. 